\title{
Trends in Management of HIV/AIDS Clients in a Tertiary Health Facility in South West Nigeria
}

\author{
Article by Amenkhienan Ibidun Florence \\ Texila American University Guyana \\ E-mail: ibidunamen@gmail.com
}

\begin{abstract}
Introduction: The scourge of HIV/AID has negative impact on human existence. Global trends in HIV prevalence are alarming despite the use of potent antiretroviral medications for many decades. The impact on individual in a society and communities at large is a public health concern and all hands should be on deck to curb this social menace. The big question is what more can we do to help get to Zero? The objective of this study was to reflect on the trends in HIV care in a tertiary health facility in South West Nigeria and compare with what is obtainable globally and elucidate what more can be done now, to safeguard the future of the next generation.

Methods: In this study, secondary data was used. Monthly records of seropositive patient enrolled in ART care for 10 consecutive years were retrieved. Including routine clinic visits, and wards admissions. A review of monthly records of seropositive clients' (adult, paediatric and antenatal clinics) enrolled in ART programs of Federal Medical Centre Owo Ondo Nigeria was conducted. Data analysis was done using SPSS version 22.0.

Result: Total number of seropositive clients enrolled between the years 2006 to 2015 in the adult ART clinic is 3844 with a monthly average of 344.4 clients. Total number of male that attended ART clinic was 1782 (46.5\%) while total number of female was 2058 (53.5\%). Prevention of Mother to Child Transmission (PMTCT) the total of pregnant women tested from year 2008 to 2015 is 19,641 but only 3530 (17.97\%) were seropositive. Highest enrollment occurred in year 2008 which was 857(22\%), with lowest enrollment occurring in 2006 which was 251(6.5\%). Therefore, showing decrease in enrolment over the years. A total of 225 clients were transferred out, 19.5\% were lost to follow up. However, total pregnant women tested from year 2008 to 2015 is 19,641 out of which 3530 (18\%) were seropositive. There is an appreciable decline in the incidence of HIV/AIDS among pregnant women from 2008 till 2015, from 884 (25\%) to 173 (4.9\%). The prevalence of seropositive pregnant women have reduced from $7.95 \%$ in 2008 to $0.9 \%$ in 2015.

Conclusion: Early enrolment in ART programme will ensure access to drugs and help safeguard the future. Adequate counseling will help form good habits and thereby reduce the spread of HIV. The use of antiretroviral therapy is significant in reducing morbidity and mortality in HIV positive clients especially in developing countries.
\end{abstract}

Keywords: Trends, Client, Seropositive, Antiretroviral.

\section{Introduction and background}

HIV/AIDS remains a major global public health concern. The fight against it requires that all hands be on deck. HIV/AIDS is a great scourge that has negatively affected our society more than anything else in history. The global trend of the virus revealed that 38.1 million people have become infected with HIV and 25.3 million people have died of AIDS-related illnesses since 2000 [1]. In the year 2014, an estimated 36.9 million people were living with the virus (2.6 million children inclusive), with a global HIV prevalence of $0.8 \%$ [2]. Majority of this people reside in the developing nations. Deaths from AIDSrelated illnesses amounted to 1.2 million people. A shocking revelation is that 25.8 million people harboring the virus reside in Sub Sahara Africa, which accounts for $70 \%$ of the global total 
DOI: $10.21522 / \mathrm{TIJPH} .2013 .05 .04 . \mathrm{Art} 024$

ISSN: $2520-3134$

[1].Surprisingly, only 54\% of all people living with HIV know that they have the virus [3]. New HIV infections amounted to approximately 2 million out of which 220,000 were children. However, these childhood infections were due to vertical transmission which occurring in-utero, during child birth and breast feeding [1]. Despite these challenges, new global efforts have meant that the number of people receiving HIV treatment has increased dramatically in recent years, particularly in resource-poor countries [3].

As of March 2015, 15 million people living with HIV were receiving antiretroviral treatment (including 823,000 children) - representing $41 \%$ of those in need. 13.5 million Of these people were in low- and middle- income countries [4].

Of all individuals, living with HIV worldwide, $9 \%$ of them reside in Nigeria [5]. Although HIV prevalence among adults is remarkably small (3.2\%) compared to other sub-Saharan African countries such as South Africa (19.1\%) and Zambia (12.5\%), the size of Nigeria's population means that there were 3.2 million people living with HIV in 2013[6] . Nigeria, together with Uganda and South Africa, account for almost half of all annual new HIV infections in sub-Saharan Africa. This is despite achieving a 35\% reduction in new infections between 2005 and 2013 [5]. Approximately 210,000 people died from AIDSrelated illnesses in Nigeria in 2013, which is $14 \%$ of the global total [5 6 7]. Since 2005, there has been no reduction in the number of annual deaths, indicative of the fact that only $20 \%$ of people living with HIV in Nigeria are accessing antiretroviral treatment (ART) [5, 7, and 8].

In Ondo state of Nigeria, Sentinel surveillance data amongst pregnant women in 2008 showed that the prevalence of HIV/AIDS was $2.4 \%$ [9] Though, over the years, the state has recorded prevalence of $2.9 \%$ in $1999,6.9 \%$ in 2001, $2.3 \%$ in 2003 and $3.2 \%$ in 2005 [9].However, the geographical distribution of HIV by states shows a prevalence of $2.1-4.0 \%$ [10]

In Owo Local Government area of Ondo the state, scanty data exist on prevalence of HIV/AIDS due to lack of record keeping. Encouraging HIV testing among the indigene population to ensure everyone knows their HIV status is a key to any informed strategic plan. Without knowing the extent of how many people are living with HIV it is hard to mitigate new infections and provide HIV treatment to all.

Federal Medical Centre Owo is a tertiary hospital in Owo Local Government Area in Ondo state, located in the south west geopolitical zone. It was established in 1989.The general mandate given to all the Federal Medical Centre within the framework of the laws establishing the center is to provide qualitative, affordable, specialized/tertiary level hospital care to the citizenry and to ultimately decrease the burden of diseases in the communities, through provision of prompt and emphatic preventive, curative and rehabilitative services [11]. FMC Owo is the First site for HIV/AIDS care in the whole south west region of the country as far back as 2006 and has worn many prices for outstanding performances, notably is the award for recognition for outstanding contribution to HIV service in Nigeria, 2010 FHI LAMIS Excellence Award in recognition as Best Treatment Evaluation Centre.

HIV disease classification and staging systems are critical tools for tracking and monitoring the HIV epidemic and for providing clinicians and clients with essential information about HIV disease stage and clinical Prevention. The stages of HIV progression includes: Stage 1- Window period, Stage 2Asymptomatic period, Stage- 3 Symptomatic period and Stage 4- Full blown AIDS [12, 13]. The three major modes of transmission includes sexual, parenteral and mother to child transmission. Kissing, hugging, eating together, working in the same office, using the same toilet with infected person cannot transmit HIV. Abstinence from sex if unmarried, being faithful to one's marital partner, consistent and condom use in specific situations, desist from sharing skin piercing or cutting devices, empowerment through education, free testing and PEP - Post Exposure Prophylaxis are well recognised preventive measures $[14,15]$.

The types of HIV/AIDS care services available in FMC Owo includes: Adult HIV care, Prevention of Mother to Child Transmission (PMTCT), pediatric HIV management, HIV Pharmacotherapy and Post Exposure Prophylaxis (PEP), Gene Expert test and DOTS for TB co- infection. As a public health care Centre, there is also provision for outreach programs for community awareness, sensitization and 
mobilization. Like the celebration of World AIDS Day since 2010 till date. All the activities of FMC Owo are in line with the national guide lines and policies on HIV/AIDS.

\section{Methods}

\section{Study environment}

Owo is an ancient city located in the South-Western part of Nigeria, in Owo local government area of Ondo State, with an estimated population of two hundred and seventy-three thousand, two hundred and twenty-six $(273,226)$. Owo is one of the ancient towns of the Yoruba people. Other tribes living in Owo are mostly Ibos and Ebiras. Most occupations among the indigene are small scale subsistence farming, petty trading and manual work. Owo is located about $350 \mathrm{~km}$ from Lagos and 50km from Akure, the state capital and is at a major intersection of roads leading to Benin and Abuja.

\section{Study site}

The Federal Medical Centre (FMC) Owo is one of the pioneers FMCs in Nigeria; it was established in 1993 by the Federal Government. FMC Owo provides tertiary healthcare services in Ondo State. The hospital is a 300 bedded hospital with staff strength of 1,325 out of which doctors and nurses constitute about 500. FMC Owo is one of the two hospitals providing tertiary care in Ondo State and has the largest volume of patient attendance in the state. The Centre serves to provide healthcare for the people in the catchment areas which comprise the entire Ondo State and neighboring Osun, Ekiti, Edo, Kogi and Lagos States. The hospital provides services in different specialties such as Family Medicine, Internal Medicine, Surgery, Obstetrics and Gynecology, Community Health, Dentistry, Ophthalmology

\section{Data collection instrument and procedure}

In this study, secondary data was used. Medical records of seropositive patient enrolled in ART care for 10 consecutive years (2006-2015) were retrieved. Including routine clinic visits, and wards admissionns. Treatment outcome were identified. A revive of monthly records of seropositive clients' (adult, paediatric and antenatal clinics) enrolled in ART programs of Federal Medical Centre Owo Ondo Nigeria was conducted. Data were analysed with SPSS version 22.0.

\section{Results}

\section{Statistics of HIV care in FMC owo}

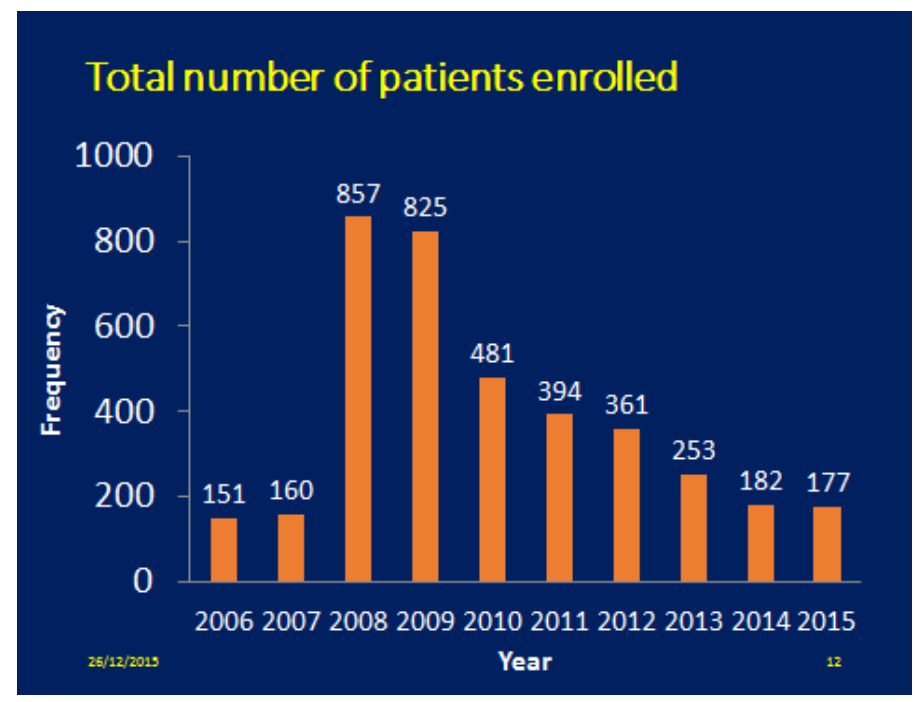

Figure 1. Shows the total number of adult clients enrolled from year 2006- 2015. 
DOI: $10.21522 / \mathrm{TIJPH} .2013 .05 .04 . \mathrm{Art} 024$

ISSN: $2520-3134$

Total number of clients enrolled is 3844 with a mean of 384.4 per year .Highest enrollment occurred in year 2008 which was 857(22\%), with lowest enrollment occurring in 2006 which was 251(6.5\%).

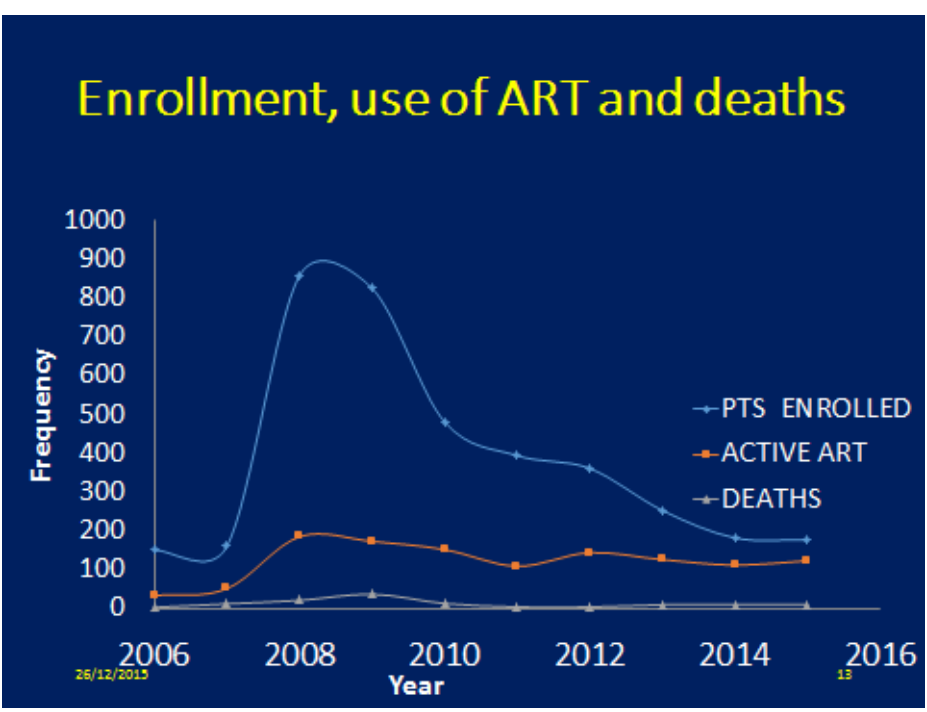

Figure 2. Shows the trend in enrollment, the use of active ART and deaths that occurred from year 2006 to 2015.

There is a slow graduation in the enrollment trend from 2006 to 2007 before attaining a sharp increase to $857(22.3 \%)$ in the year 2008. This was maintained till 2009 being 830(21.6\%) before reducing gradually to $182(4.7 \%)$ and almost same in 2015 .

The use of ART showed an increase in 2008 and has being maintained till 2015. There was a correlation of enrolment and ART use at the onset of the programme simultaneously until 2011. There was an increase before it maintained a balance through the years 2012 to 2015.

Deaths since onset of ART use and patient enrollment has maintained a low level remaining below 30 clients per month. However, no deaths were recorded in the year 2006, 2011 and 2012. Reasons for death in 2008-2012 could be attributed to late presentation and co-morbidity.

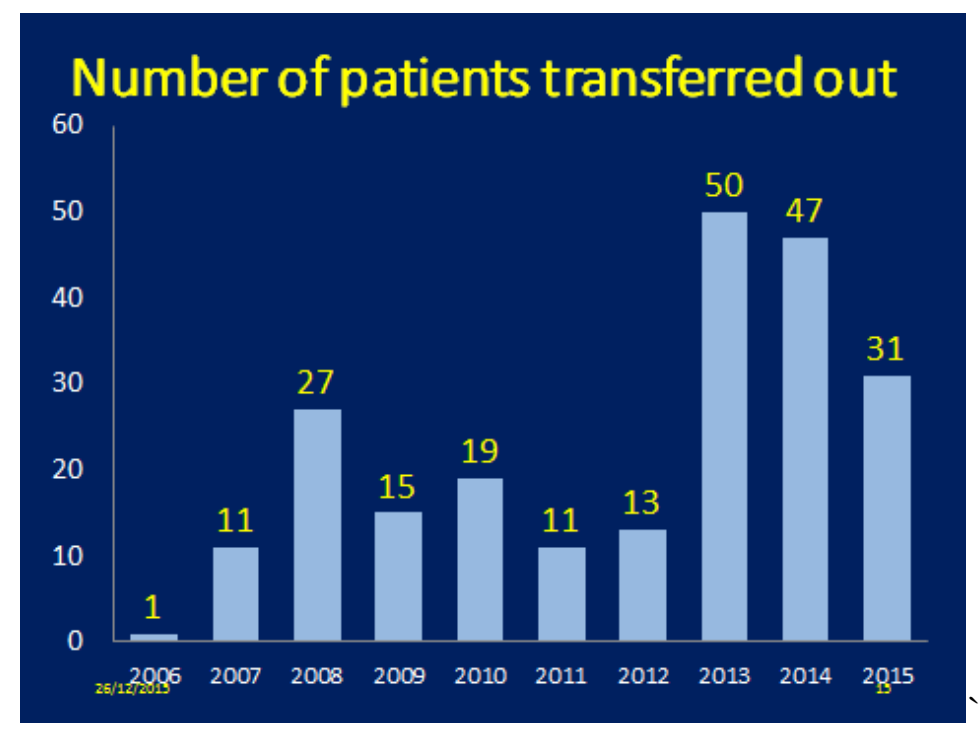

Figure 3. Shows the number of clients transferred out.

Total of 225 clients was transferred out, $19.5 \%$ of the total clients enrolled. 
In 2006, only one patient was transferred out but an increase sustained through 2007 to 2008(11 clients to 27 clients). The maximum number of transfer was in 2013, 50 clients, (19.8\%) of enrolled clients.

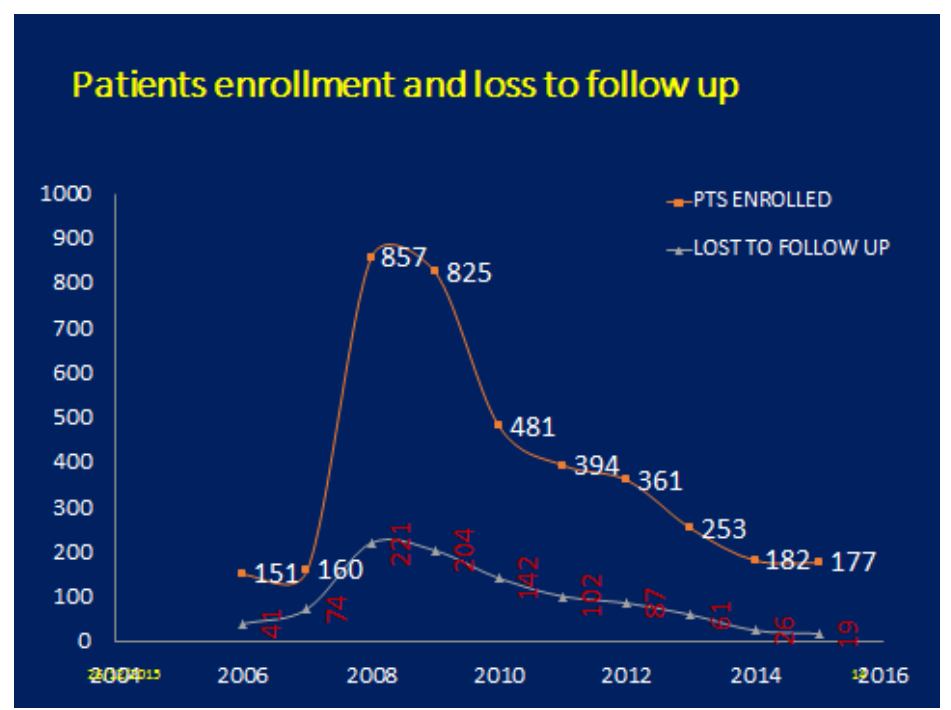

Figure 4. Shows the clients enrolled and those lost to follow up.

In 2008, when enrollment seems to be at its peak (857), a total number of 221(25.8\%) clients were lost to follow up indicating the highest. There is a significant decline in the trend till 2015 19clients. (2.2\%).

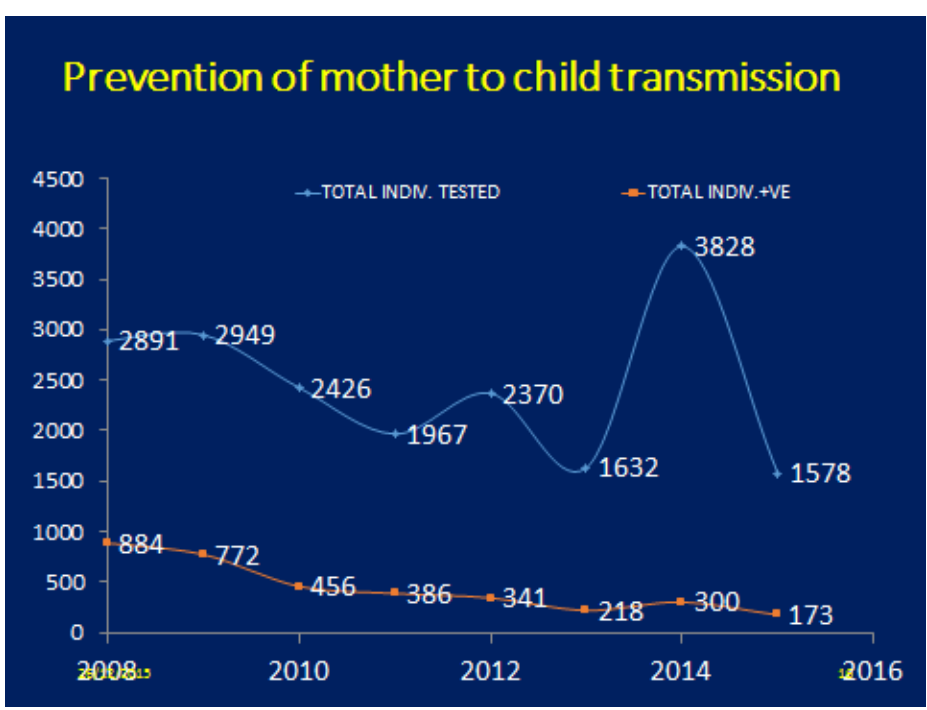

Figure 5. Shows total pregnant women tested and total pregnant mothers who were positive from 2006-2015.

The total of pregnant women tested from year 2008 to 2015 is 19,641. The year 2014 had the highest number of pregnant women tested 3,828(19.5\%). Total number of positive pregnant women $3530(18 \%)$ of the pregnant women tested.

However, there is an appreciable decline in the incidence of HIV/AIDS among pregnant women from 2008 till date from 884 ( $25 \%$ ) to 173 (4.9\%). 
DOI: $10.21522 / \mathrm{TIJPH} .2013 .05 .04 . \mathrm{Art} 024$

ISSN: $2520-3134$

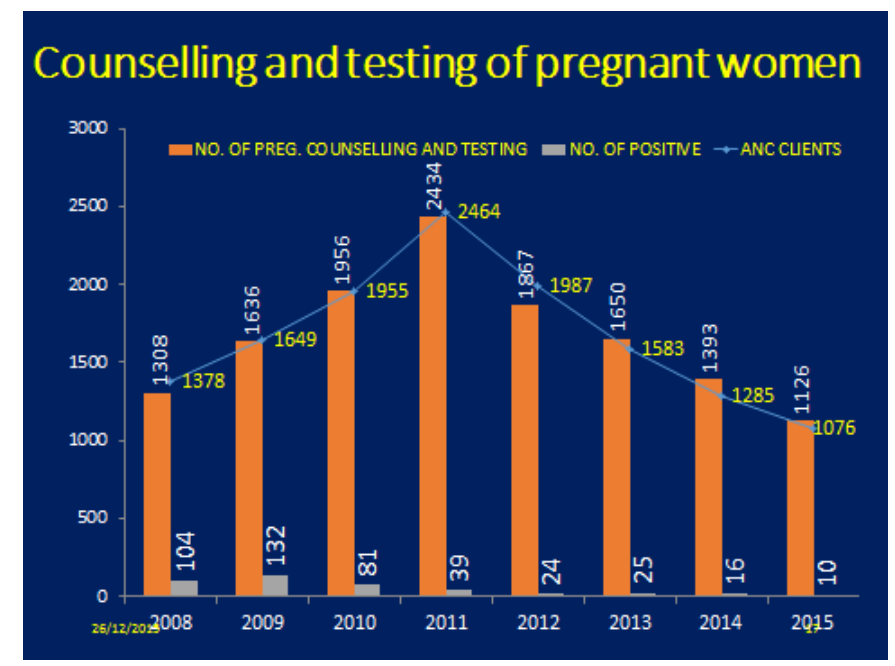

Figure 6. Shows the number of counseled and tested pregnant women, the numbers of HIV positive pregnant women and the ANC clients. A total of 13,370 were offered HIV counseling and testing. Out of which 431(3.2\%) were positive. The total number of ANC clients was 13,327 pregnant women. The prevalence seropositive pregnant women have reduced from $7.95 \%$ in 2008 to $0.9 \%$ in 2015 .

\section{Discussion}

Total number of clients enrolled was 3844 adult clients .Highest enrollment occurred in year 2008 which was 857(22\%), with lowest enrollment occurring in 2006 which was 251(6.5\%). The use of ART showed an increase in 2008 and has been maintained till 2015. There is a correlation of enrolment and ART use at the onset of the programme simultaneously until 2011. There was an increase before it maintained a balance through the years 2012 to 2015 . Enrollment will provide better access to healthcare coverage and more health insurance options. [16].

Deaths since onset of ART use and patient enrollment has maintained a low level remaining below 10 clients per month. However, no deaths were recorded in the year 2006, 2011 and 2012. Reasons for death in 2008-2012 could be attributed to late presentation and co-morbidity. A research done revealed that ART drugs in combination gave patient and scientist new hope for fighting the epidemic and has significantly improved life expectancy to decades rather than months. [17].

Decrease in enrollment since 2009 till date is because more sites have been established for HTC so clients have been transferred out to those sites. Moreover, transfer of clients leads to decrease in enrollment in 2006, just one patient was transferred out but an increase sustained through 2007 to 2008(11 clients to 27 clients). The maximum number of transfer was in 2013(50 clients) which is equivalent to $19.8 \%$ of enrolled clients. . There is a significant decline in the trend till 2015, 19client (10.7\%). The barriers to enrollment of clients in ART differ in location, ranging from few facilities to cultural appropriate and efficient practices for allocating ART to reach the rural and marginalized populations. Other factors like financial and logistics barriers might be responsible (transportation risk and costs) [18].

In 2008, when enrollment seems to be at its peak (857), a total number of 221(25.8\%) clients were lost to follow up indicating the highest. There is a significant decline in the trend till 2015 19client (10.7\%). This is due to people moving away from the programme and a few deaths have occurred. There is Mother and Child care in Ondo State hospital and SSH, Akure where clients can receive ART and care (Decentralization of services.)

Total number of positive pregnant women was 3530 (18\%) of the pregnant women tested. However, there is an appreciable decline in the incidence of HIV/AIDS among pregnant women from 2008 till 2015, from $884(25 \%)$ to $173(4.9 \%)$. Female education, empowerment and ability to negotiate safer sex practices are adduced to this reduction in positive cases. In many countries, there have been a decrease in 
number of pregnant women receiving ART including Botswana, Chad, Ghana, South Africa, Zambia; Lesotho due to better monitoring systems, shortage of staff and accessibility might be responsible [19].

\section{Adult HIV care in FMC owo}

HIV disease classification and staging systems are critical tools for tracking and monitoring the HIV epidemic and for providing clinicians and clients with essential information about HIV disease stage and clinical Prevention. The stages of HIV progression includes: Stage 1- Window period, Stage 2Asymptomatic period, Stage- 3 Symptomatic period and Stage 4- Full blown AIDS [12, 13] The three major modes of transmission includes sexual, parenteral and mother to child transmission. Kissing, hugging, eating together, working in the same office, using the same toilet with infected person cannot transmit HIV. Abstinence from sex if unmarried, being faithful to one's marital partner, consistent and condom use in specific situations, desist from sharing skin piercing or cutting devices, empowerment through education, free testing and PEP - Post Exposure Prophylaxis are well recognised preventive measures[14,15].

In the adult HIV care in FMC Owo, Screening is the first step into enrolment into care, as a protocol clients are enrolled through the process of voluntary counseling and testing, usually this is client initiated, although Provider-initiated Testing and counselling is available depending on the prevailing circumstances. Furthermore, every service provider is expected to offer their client/clients the opportunity of knowing their status. Entry points for adults take place mainly at HEART TO HEART, while others come in through Family Planning Unit, TB/ DOT centre, PMTCT Services i.e. antenatal unit, referral and outreaches .It is projected that in the nearest future, through integration of services, screening shall be offered at every service delivery point in the hospital.

The next step is to determine clients' eligibility for ART [Anti- Retroviral Therapy]. Staging is done by assessing each patient for co morbidity especially Tuberculosis. The $\mathrm{CD}_{4}$ count primarily determines the management modality. ART is initiated in all individuals with severe or advanced HIV clinical disease (WHO clinical stage 3 or 4 ) and individuals with CD4 count $\leq 350$ cells $/ \mathrm{mm} 3$. Currently the National guideline on ART recommends 500cell $/ \mathrm{mm}^{3}$ : as the baseline for ART commencement in adults [20]. This is what we practise in this centre. It is hoped that all clients diagnosed positive will commence ART in the near future as practised in developed countries (WHO recommendation). During continuum of care there is assessment for opportunistic infection at every clinic visit and initial adherence counseling is reinforced. Cotrimoxazole is given for prophylaxis against opportunistic infections at every visit if there are no contraindications. [20]

Following commencement of HAART [Highly Active Antiretroviral Therapy] clients are seen at 2 weeks interval and later every two months. Disclosure especially to spouse is encouraged but never forced on clients.

\section{Prevention of mother to child transmission (PMTCT)}

Prevention of Mother to Child Transmission (PMTCT) of HIV. Mother to child transmission can occur during pregnancy, through the process of labor and delivery as well as breastfeeding. Most children less than 15 years of age, living with HIV acquire the infection through MTCT [1].

Nigeria has the $2^{\text {nd }}$ highest number of HIV infected pregnant women globally (after South Africa) contributing 30\% PMTCT gap burden globally (13\% Coverage; 2009). 80\% of HIV transmission in Nigeria is heterosexual, and a notable fact is that women are more at risk to contracting the virus than men, due to anatomical, socio-economical cultural and religious factors associated with the female gender. 3.4\% of childbearing women in Nigeria are HIV-positive (2013 survey).

Elements for comprehensive approach to prevent HIV infection, in infant and young children as practiced in FMC Owo includes: (1) Primary prevention of HIV infection, (2) Prevention of unintended pregnancies among women infected with HIV, (3) Prevention of HIV transmission from women infected with HIV to their infants, (4) Provision of treatment, care, and support to women infected with HIV, their infants and their families. According to researches done worldwide, Amongst 100 infants born to HIV- 
DOI: $10.21522 / \mathrm{TIJPH} .2013 .05 .04 . \mathrm{Art} 024$

ISSN: $2520-3134$

infected women who breastfeed, without any interventions,5 -10 infants would be infected during pregnancy, about 15 infants will be infected during labor and delivery, while 5-15 infants will contract the virus during breast-feeding. 25-40 infants will be HIV-infected; nonetheless 60 to 75 infants will not be HIV-infected [22].

Risk factors for MTCT are the pregnancy itself, labor and delivery, as well as breastfeeding. During pregnancy, high maternal viral load, infections, STI's, malnutrition, and hemorrhage can predispose the child HIV. However, during labor and delivery, factors such as high maternal viral load, prolonged rupture of membranes, chorioamnionitis, invasive delivery procedures, instrumental delivery, episiotomy, lacerations the first infant in multiple births and fetal genetic characteristics. Furthermore, concerning breastfeeding, high viral load, the duration of breastfeeding, breast fissures infections poor maternal nutrition and oral disease in infants are documented ways of MTCT.

HIV counseling and testing HCT is done at every point of contact of pregnant woman with the health facility even in the labor and obstetrics wards. Pregnant women who come for anti natal care booking are offered HIV counseling and testing (HCT) during group health talk with the option of right to refuse (optout approach). Women who consent to test are tested privately (confidential testing) and result given same way. Post-test counseling is thereafter done. Confidentiality is maintained from the stage of testing and throughout the care of the woman.

For those who are HIV positive, clinical staging of the disease (WHO criteria) and appropriate laboratory tests are then done as baseline and as follow-up. Anti-retroviral (ARV) drugs are given in addition to routine obstetric care. Testing, laboratory investigations and drugs are free in FMC Owo due to support by partners (FHI, Equitable Health Initiative) and the Federal Government of Nigeria.

Eligibility criteria for ART or ARV prophylaxis in HIV infected pregnant women 2014 guidelines

\begin{tabular}{|l|l|}
\hline CD4 Cell count available & \\
\hline $\mathrm{CD}_{4} \leq 500$ cells $/ \mathrm{mm}^{3}$ & $\mathrm{CD}_{4}>500$ cells $/ \mathrm{mm}^{3}$ \\
\hline $\begin{array}{l}\text { ART } \\
\text { Regardless of clinical stage }\end{array}$ & ART \\
If symptomatic( stage 3 or 4$)$
\end{tabular}

In the drug treatment and care during labor, different treatment options have been used in the past, but currently any HIV-positive woman who is pregnant or breastfeeding irrespective of the CD4 count or clinical stage should be on triple ARV i.e. HAART (2014 National Guidelines). Appropriate modification of care in labor is done including avoiding invasive procedures to reduce the risk of MTCT. Special care including chlorhexidine birth is given to the HIV exposed baby. Mothers are advised to exclusively breastfeed their babies exclusively for at least 6 months.

All HIV-exposed babies are given nevirapine for 6 weeks irrespective of the feeding practice and the extension of nevirapine prophylaxis beyond 6 weeks is done in situations when the mother is breastfeeding and not on HAART (Nevirapine given until 1 week after cessation of breastfeeding). Also in situations where the mother has started breastfeeding the child before she was commenced on HAART (The child is placed on Nevirapine until 12 weeks after maternal commencement of HAART. It is one of the components of comprehensive care for HIV/AIDS. PMTCT is an effective intervention of reducing transmission of HIV to newborns it can reduce risk of transmission in newborns to < $2 \%$ [23]. 


\section{Peadiatric HIV management in FMC owo}

Pediatric HIV management started in April 2000, with recurrent training and retraining of officers over the years to provide quality care. Routine clinic services (exposed and infected clients), ward admission and care for emergency cases are well managed at the pediatric department.

Statistics of patient from 2006 to 2015

\begin{tabular}{|l|l|}
\hline Patient & Frequency \\
\hline Patient enrolled into care(1- 14 Years) & 250 \\
\hline Exposed babies enrolled into care & 430 \\
\hline Clients on ART & 240 \\
\hline Exposed babies that were started on ART & 2 \\
\hline Clients transferred out & \multicolumn{2}{|l|}{} \\
\hline Exposed & 3 \\
\hline ART & 20 \\
\hline Patient lost to follow up & \\
\hline Exposed & 10 \\
\hline ART & 72 \\
\hline Total number of patient who died & \\
\hline Exposed & 2 \\
\hline ART & 2 \\
\hline
\end{tabular}

Entry points of care into the pediatric HIV management are through the labor wards, children emergency unit and clinics (incidental findings, referrals)

\section{Management of pediatric patient}

1) Eligibility criteria for drugs:

a) Clients less than 5years, irrespective of CD4 count or \%.

b) Clients that is 5 years or more with CD4 $<500$ cells $/ \gamma \mathrm{L}$

2) Counseling (parents /Care Giver) -3 adherence counseling sessions

3) Baseline investigations; FBC, LFT, E/U/Cr, CD4 count

4) Commence HAART Prophylaxis for the exposed babies

Co-trimoxazole

Nevirapine

5) Regular follow up.

\section{Medical laboratory testing (adult and children)}

Medical laboratory testing of HIV in adult and children is an approach to patient's care, important steps in the testing process includes;

1. Provide pre-test counseling

2. Obtain test sample (Blood/Serum

3. Process sample on site or through laboratory

4. Obtain results: Keep confidential

5. Give the test result to client in person/ requesting doctor

6. Provide post- test counseling, support, and referral.

The types of tests available at the Heart to Heart laboratory are

1) HIV diagnostic tests (Rapid tests, ELISA test and Western blot.)

2) Measurement of CD4 levels to determine who qualifies for treatment

3) Monitoring of CD4 and viral load levels to ensure that treatment is still effective and to monitor immune reconstitution 
DOI: $10.21522 / \mathrm{TIJPH} .2013 .05 .04 . \mathrm{Art} 024$

ISSN: $2520-3134$

4) Chemistry and hematology test (FBC, LFT, RFT, RBS e.c.t)

5) Gene Xpert for TB co infection.

\section{Rapid tests}

- Qualitative tests.

- Based on chromatography or concentration diagnose HIV 1 and or 2

- Requires very small volumes.

- No special equipment

- No laboratory skill level and results are obtained within 15-20minutes

- Window period" of 6 weeks to 6 months may produce false negative results.

\section{Enzyme immuno-sorbent assays (ELISA)}

- Quantitative tests

- Based on color change and sensitivity

- Diagnoses HIV 1 and 2

- Needs large volumes

- Special equipment

- High laboratory skill level

- Result may take days.

\section{Western blot/Line immunoassay}

- Has high specificity

- Expensive

- Requires special skills to perform

- Only used for confirmation of positive results.

Others: P24, PCR, NAAT (not always available)

\section{Testing in children}

The "Rapid tests" /EIA are reliable after 18 months Western blot analysis.

DNA PCR (Gold standard) usually done anytime from 6 weeks of age or 6 weeks after cessation of breastfeeding.

P24 antigen is the earliest diagnostic test, which can detect as early as 3 weeks after infection before antibodies have formed, it is complex and expensive

\section{HIV/AIDS pharmacology in FMC owo}

The ART pharmacy is a special section of the main pharmacy where clients collect their free ARDs. From available records, an average monthly coverage of about 600 clients occurs (new and old for refill inclusive) a monthly range of 12-17 clients are also commenced on HAART for the first time. 


\begin{tabular}{|l|l|l|l|}
\hline $\begin{array}{l}\text { Classes of ANTI } \\
\text { retroviral drugs }\end{array}$ & $\begin{array}{l}\text { Nucleoside/Nucleo } \\
\text { tide Reverse } \\
\text { Transcriptase } \\
\text { Inhibitors } \\
\text { (NRTIs) }\end{array}$ & $\begin{array}{l}\text { Non- } \\
\text { Nucleoside/Nucleo } \\
\text { tide Reverse } \\
\text { Transcriptase } \\
\text { Inhibitors } \\
\text { (NNRTIs) }\end{array}$ & $\begin{array}{l}\text { Protease } \\
\text { Inhibitors (PIs) }\end{array}$ \\
\hline MODE OF ACTION & $\begin{array}{l}\text { Inhibits reverse } \\
\text { transcriptase } \\
\text { enzyme of the HIV } \\
\text { from converting } \\
\text { viral RNA to viral } \\
\text { DNA incorporable } \\
\text { into host DNA }\end{array}$ & $\begin{array}{l}\text { Inhibits reverse } \\
\text { transcriptase } \\
\text { enzyme of the HIV } \\
\text { from converting } \\
\text { viral RNA to viral } \\
\text { DNA incorporable } \\
\text { into host DNA }\end{array}$ & $\begin{array}{l}\text { Inhibits protease } \\
\text { enzymes from } \\
\text { advancing the } \\
\text { virions generated to } \\
\text { infective HIV }\end{array}$ \\
\hline $\begin{array}{l}\text { AVAILABLE } \\
\text { EXAMPLES IN THE } \\
\text { PHARMACY }\end{array}$ & $\begin{array}{l}\text { ZIDOVUDINE } \\
\text { (AZT) } \\
\text { LAMIVUDINE } \\
\text { (3TC) } \\
\text { ABACAVIR } \\
\text { (ABC) } \\
\text { TENOFOVIR } \\
\text { (TDF) }\end{array}$ & $\begin{array}{l}\text { NEVIRAPINE } \\
\text { (NVP) } \\
\text { EFAVIRENZ } \\
\text { (EFV) }\end{array}$ & $\begin{array}{l}\text { LOPINAVIR/RIT } \\
\text { ONIVIR(LPV/r) } \\
\text { ATAZANAVIR/R } \\
\text { ITONIVIR } \\
\text { (ATA/r) }\end{array}$ \\
\hline
\end{tabular}

First line regimen

$$
\begin{aligned}
& \text { Adult first line } \\
& \text { Preferred - TDF/3TC/EFV* } \\
& \text { Alternate - AZT or ABC/3TC/NVP or EFV } \\
& \text { Pediatric first line } \\
& \text { Preferred - AZT/3TC/NVP (0-3 Years of age) } \\
& \text { Alternate - ABC/3TC/NVP (0-3 Years of age) } \\
& \text { Preferred - AZT/3TC/EFV (3-10years of age) } \\
& \text { Alternate - ABC/3TC/EFV (3-10years of age) }
\end{aligned}
$$

\section{Pediatric first line}

\section{Adult second line}

Preferred - AZT/3TC/ATV/r or LPV/r

Alternate - TDF/3TC/ATV/r or LPV/r

\section{Pediatric second line}

\section{$\mathrm{ABC} / \mathrm{AZT}+3 \mathrm{TC}+\mathrm{LPV} / \mathrm{r}$}

*LPV/r- usually preserved for second line replacement of NNRTIs (NVP/EFV)

Use of second line regimen: Notable reason why clinician and pharmacist(switch committee) in FMC Owo do switch to second line drugs is treatment failure which could be as a result of insufficient potency of the ARV regimen, insufficient drug levels, Pre-existing viral drug resistance, development of drug resistance mutations, poor prescribing or dispensing, inadequate/inconsistent drug supply.

\section{Regimens for special categories of adults/adolescents}

Women on ART and gets pregnant 
DOI: $10.21522 / \mathrm{TIJPH} .2013 .05 .04 . \mathrm{Art} 024$

ISSN: $2520-3134$

ZDV (Zidovudine) + 3TC (lamivudine) + EFV (Efavirenz)/NVP

Considerations are made to switch TDF in place of ZDV if there is evidence of anemia. NVP is used only if CD4 cell count is $<350$ cell $/ \mathrm{ml}$. However, monthly CD4 count test is recommended to

Efavirenz not used in first trimester

With NNRTIs failure, LPV/r or SQr/r or ATA/r is used.

Women diagnosed during HTC and found pregnant are commence on ART immediately regardless of CD4 count or WHO clinical stage

Note: for pregnant women; test and treat!

\section{Infants born to HIV positive mothers}

- Single dose of NVP should be given within 72hours after birth

- If breastfed, should continue with NVP daily dosing till 1week after weaning

- If not breastfed, should continue the daily dosing of NVP for 6weeks after delivery.

- Baby $<2.5 \mathrm{~kg}=10 \mathrm{mg}$ daily and $>2.5 \mathrm{~kg}=15 \mathrm{mg}$ daily $\mathrm{NVP}$

\section{Post exposure prophylaxis (PEP)}

- PEP consists of 2-3 antiretroviral medications and must be taken for 28 days

- by health care workers who have been exposed to HIV-infected fluids on the job (occupational)or anyone who may have been exposed through unprotected sex, needle-sharing injection drug use, or sexual assault.(non-occupational)

- The recommended PEP regimens are:

- For adults:

- TDF/AZT+3TC (in low risk exposed clients)

- Above with LPV/r (in high risk exposed clients)

- For children

- $\mathrm{AZT}+3 \mathrm{TC}+\mathrm{LPV} / \mathrm{r}$

- Mono -therapy no longer used

\section{Conclusion}

HIV/AIDS is a crisis for global public health attention, and not only the health sector. The disease impedes on development, thus affecting the socio-economic gains that the developing countries are aspiring to attain because it constitutes a threat to human race in terms of security and development by affecting the Health Related Millennium Development Goals (MDG1, 4, 5, 6,7and 8) and Sustainable Development Goals. From the trends of events highlighted in the study, early enrolment in ART programme will ensure access to drugs and help safeguard the future. Adequate counseling will help form good habits and thereby reduce the spread of HIV. The use of antiretroviral therapy is significant in reducing morbidity and mortality in HIV positive clients especially in developing countries. Worldwide it is noted that prevalence of seropositive pregnant women is reducing, also mother to child transmission is also reducing as seen in many studies done across the globe. It could therefore be projected that mother to child transmission can reach zero level. The use of efficient patient tracking system can be used to combat the problem of defaults and loss to follow-up.

\section{Recommendation}

Encouraging HIV testing among the indigenes of Owo, and the nation at large to ensure everyone knows their HIV status is the key to any informed strategic plan. Without knowing the extent of how many people are living with HIV it is hard to mitigate new infections and provide HIV treatment to all. PMTCT should be embraced by all government, it can actually help the world in" getting to Zero" in terms of new infection, transmission and discrimination. 


\section{References}

[1]. Bulletin of World Health Organization. Understanding the modes of transmission model of new HIV infection and its use in prevention planning. Kelsey Case ${ }^{\mathrm{a}}$, Peter D Ghys ${ }^{\mathrm{b}}$, Eleanor Gouws ${ }^{\mathrm{b}}$.

[2]. Centre for Disease control and Prevention. 1993 revised classification.

[3]. Dieffenbach CW, Fauci AS. Thirty years of HIV and AIDS, future challenges and opportunities. Ann Interm Med 2011; 154:766-771.

[4]. HIV Classification: CDC and WHO staging systems Published Date: April 30, 2014.

[5]. HIV CLINICAL RESOURCES. HIV Prophylaxis Following Occupational Exposure. Updated October 2014.hivguidelines.org.

[6]. Infectious Diseases society of America. Antiretroviral therapy may prevent HIV transmission from breastfeeding mothers to infants, Science Daily July 31, 2005.

[7]. NATIONAL GUIDELINES FOR HIV/AIDS TREATMENT AND CARE IN ADOLESCENT AND ADULTS FMOH ABUJA-NIGERIA OCTOBER 2010.

[8]. Mattew fox, Factors influencing Enrollment in Treatment for HIV/AIDS in Zambia, Donor/Finder CDC, 20072009.bu.edu/cghd/our.work/project.

[9]. Nigeria 2013 HIV Survey. Last viewed on 01/12/15

[10]. Nigeria HIV Fact Sheet - US Department of State.

[11]. Nigeria National Agency for the Control of AIDS (2014) 'Countries Progress Report 2014'

[12]. Ondo State Ministry of Health HIV AIDS Control programme: www.ondostatemoh.gov.ng/hiv-aids.php. Last viewed on 21/12/15

[13]. "Reviewed Charters for Federal Medical Centres pp 1". Servicom Charters. Servenigeria.com. Retrieved 13 June 2011.

[14]. THE WORLD BANK: worldband.org/indicator/SH.HIV.ARTC.2S?

[15]. The Affordable care Act and HIV/AIDS. http://www.aids.gov/federal-resources. Last viewed on 21/12/15.

[16]. UNAIDS (2015) 'Fact sheet: 2014 statistics'. Last viewed on 20/12/2015

[17]. UNAIDS (2015) 'How AIDS Changed Everything'.

[18]. World Health Organization (WHO) (2015) 'HIV/AIDS'

[19]. UNAIDS and The Henry J. Kaiser Family foundation 2014 'financing the Response to HIV in low and MiddleIncome Countries: International Assistance from Donor Governments in 2013'.

[20]. UNAIDS (2014) 'The Gap Report'.

[21]. UNAIDS (2014) 'Epidemiology Fact Sheet on HIV and AIDS in Nigeria'.

[22]. UNAIDS (2014) 'the Gap Report 2014'. Last viewed on 10/12/15

[23]. WHO/HIV/AIDS Mother-to-child transmission of HIV latest updates July 2015. 